\title{
New and Newsworthy Neurocritical Care Studies
}

\author{
Alejandro A. Rabinstein
}

Published online: 26 October 2010

(C) Springer Science+Business Media, LLC 2010

\section{Prediction of Respiratory Insufficiency in Guillain- Barré Syndrome}

Walgaard C, Lingsma HF, Ruts L, Drenthen J, van Koningsveld R, Garssen MJ, van Doorn PA, Steyerberg EW, Jacobs BC

Ann Neurol 2010;67(6):781-787

This study used a derivation cohort of 397 patients with Guillain-Barré syndrome (GBS) to identify predictors of mechanical ventilation in the first week of hospital admission and a separate cohort of 191 GBS patients to test the validity of the model constructed with the predictors previously identified. The rates of mechanical ventilation during the first week of admission were $22 \%$ in the derivation cohort and $14 \%$ in the validation cohort. The variables that made it into the model were: (1) days between onset of weakness and hospital admission (the shorter the interval, the higher the risk of requiring mechanical ventilation); (2) facial or bulbar weakness at hospital admission; and (3) Medical Research Council (MRC) sum score at hospital admission (the lower the sum score, the higher the risk of requiring mechanical ventilation). The combination of admission within 3 days of onset of weakness, presence of early facial or bulbar weakness, and more severe, generalized weakness in the extremities was most strongly associated with the requirement of mechanical ventilation during the first week of admission.

The predictive model reported in this study is simple and practical. It may be particularly useful to decide which patients need admission to the ICU for closer monitoring.

A. A. Rabinstein $(\bowtie)$

Mayo Clinic, Rochester, MN, USA

e-mail: rabinstein.alejandro@mayo.edu
Once this first decision is made, other variables need to be evaluated for early prognostication, including the results of electrophysiology and beside pulmonary function tests. It is also important to remember that the factors influencing acute respiratory insufficiency are not the same ones that determine long-term recovery (such as older age and axonal form of GBS).

\section{Improved Cognitive Outcomes with Endovascular Coiling of Ruptured Intracranial Aneurysms: Neuropsychological Outcomes from the International Subarachnoid Aneurysm Trial (ISAT)}

Scott RB, Eccles F, Molyneux AJ, Kerr RS, Rothwell PM, Carpenter K

Stroke 2010;41(8):1743-1747

This neuropsychological sub-study of 836 patients enrolled to ISAT in 8 UK centers found that nearly one-third of non-disabled (modified Rankin 0-2) survivors of aneurysmal subarachnoid hemorrhage have cognitive impairment 12 months after the acute event. The risk of cognitive impairment was much lower in patients who underwent coiling of the aneurysm than in those who were treated with neurosurgical clipping (27 vs 39\%; OR $=0.58,95 \% \mathrm{CI}$ $0.38-0.87$ ). The presence of cognitive impairment was strongly associated with lower self-reported quality of life. Cognitive impairment was not determined by the development of epilepsy (though epilepsy was also less common in the coiling group).

The results are also enlightening by showing that nearly one-quarter of patients with a modified Rankin score of 0 had evidence of cognitive impairment on neuropsychological testing, confirming that this score fails to identify cognitive deficits and explaining why so many 
patients fail to return to their previous occupation despite what seems to be a favorable functional recovery. They also reaffirm the validity of data from previous studies which indicated that the most affected cognitive domains (i.e. verbal memory, processing speed, executive skills) localize the problem to the frontal lobes, suggesting that damage from frontal lobe retraction during surgery could be responsible for the disparity in favor of coiling.

\section{Temporal and Spatial Profile of Brain Diffusion- Weighted MRI After Cardiac Arrest}

Mlynash M, Campbell DM, Leproust EM, Fischbein NJ, Bammer R, Eyngorn I, Hsia AW, Moseley M, Wijman CA Stroke 2010;41(8):1665-1672

In this study 38 scans from 33 post-cardiac arrest patients (18 with good outcome and 15 with poor outcome) were compared those of 14 controls. All scans were obtained within 8 days of the arrest (median 3.3 days). Diffusionweighted imaging differed substantially in the two outcome groups of post-cardiac arrest patients. Patients with poor outcome (Glasgow outcome score 1 or 2) had multiple areas of restricted diffusion, primarily affecting the occipital and temporal cortex and the putamen. These changes were already present in the second day, but could be absent during the first $24 \mathrm{~h}$ and only reached their peak severity 3-5 days after the arrest. Marked restricted diffusion in the white matter was noted at later intervals (6-8 days).

Meanwhile, patients with good outcome (albeit the definition of good outcome included patients with Glasgow outcome score of 3, i.e. those who awoke but remained severely disabled) showed increased diffusivity in the hippocampus, temporal and occipital lobes, and corona radiata. The authors hypothesize that this finding could represent a mild degree of early vasogenic edema in some survivors of cardiac arrest (as opposed to the cytotoxic edema seen in poor outcome patients).
The message of this study is that persistent coma from anoxic brain injury after cardiac arrest is predicted by the presence multifocal areas of restricted diffusion in the cortex and deep basal ganglia on MRI scans obtained more than between 1 day and 1 week after the arrest. The next step is to investigate what findings on MRI predict functional recovery in survivors who awaken from their coma.

\section{Effects of Tranexamic Acid on Death, Vascular Occlusive Events, and Blood Transfusion in Trauma Patients with Significant Haemorrhage (CRASH-2): A Randomised, Placebo-Controlled Trial}

CRASH-2 trial collaborators

Lancet 2010;376(9734):23-32

CRASH-2 was a massive international randomized, blinded, controlled trial comparing tranexamic acid $(1 \mathrm{~g}$ loading and then $1 \mathrm{~g}$ infused over $8 \mathrm{~h}$ ) versus placebo administered to adult trauma patients with significant hemorrhage (hypotension or tachycardia) or deemed to be at risk for hemorrhage. Enrollment took place within $8 \mathrm{~h}$ of the injury.

Mortality in this trial was $15 \%$. Over one-thirds of these deaths occurred within the first $24 \mathrm{~h}$ and also one-third were due to bleeding. The main result was that tranexamic acid reduced the risk of fatal traumatic hemorrhages. The risk of vascular occlusive events was not increased by the administration of the antifibrinolytic agent. Benefit was strong among patients treated within $3 \mathrm{~h}$ of the injury, but no longer evident when the drug was started after $3 \mathrm{~h}$.

However, it is difficult to know how to apply the results of this trial to patients with pure or predominant traumatic brain injury. Mortality from head injury did not differ between the two groups. Need for neurosurgery was also similar. Therefore, more studies are needed to examine the effect of tranexamic acid on the extent of intracranial bleeding and to confirm the safety of this medication in patients with severe traumatic brain injury. 